Published Ahead of Print on December 13, 2006 as 10.1097/01.psy.0000249734.99065.6f

\title{
Flexible Coping Psychotherapy for Functional Dyspeptic Patients: A Randomized, Controlled Trial
}

\author{
Cecilia Cheng, PhD, Feng-Chi Yang, MD, Song Jun, MD, and Jane M. Hutton, PhD
}

\begin{abstract}
Objective: This study tested the efficacy of a new psychotherapy, flexible coping psychotherapy (FCP), specifically designed for enhancing coping flexibility of patients with functional dyspepsia (FD). The design of this psychotherapy is based on the general cognitive-behavioral model and previous findings on FD. Methods: We adopted a randomized, controlled design to examine the differences between the target (FCP) and control (supportive psychotherapy [SPP]) conditions. Coping flexibility and outcome measures reported by 75 Chinese FD patients (18-65 years; 35\% men) were assessed before and 12 months after treatment. Results: Results revealed that participants who received the FCP reported an increase in coping flexibility as well as reductions in self-rated dyspeptic symptom severity (SDSS), gastroenterologist-rated dyspeptic symptom severity, and anxiety levels ( $p$ values <.01). Participants who received the SPP reported reductions in SDSS and anxiety levels $(p$ values $<.0001)$. Although both groups reported a decrease in SDSS, only the SDSS level of the FCP group was comparable to that of a healthy community sample $(p=.28)$. Conclusions: These findings suggest that FCP is a potentially effective intervention for treating FD symptoms. Its effectiveness may be attributable to the specific components of FCP rather than common psychotherapeutic factors such as emotional support and empathy per se. Key words: anxiety, coping, functional dyspepsia, psychotherapy, randomized controlled trial, stress.
\end{abstract}

$\mathbf{C F Q}=$ Cognitive Flexibility Questionnaire; DSQ $=$ Dyspeptic Symptom Questionnaire; FCP = flexible coping psychotherapy; FD = functional dyspepsia; GDSS = gastroenterologist-rated dyspeptic symptom severity; MANOVA = multivariate analysis of variance; $\mathbf{R C I}=$ reliable change index; $\mathbf{S D S S}=$ self-rated dyspeptic symptom severity; $\mathbf{S P P}=$ supportive psychotherapy; $\mathbf{S T A I}=$ StateTrait Anxiety Inventory.

\section{INTRODUCTION}

A ccording to the Rome II Diagnostic Criteria (1), functional dyspepsia (FD) is a functional problem with pain or discomfort centered in the upper abdomen for at least 12 weeks within 1 year without any structural or biochemical causes identified. The current standard treatment of FD is medical, emphasizing pharmacological interventions and dietary changes (2). It is worth noting that few drugs and dietary plans have beneficial effects, and most patients with FD are not responsive to medical treatment (see (3)).

Compared with studies evaluating medical treatment, the number of studies examining the efficacy of psychotherapy on FD is relatively scant. Although there is growing evidence indicating the therapeutic value of psychotherapy-such as supportive psychotherapy and cognitive psychotherapy-on FD (see $(4,5)$ ), a critical review of this body of research revealed that most therapists adopted therapies designed for treating other psychological problems. The rationale given to the type of psychotherapy selected was unknown. To date, no studies have adopted systematic models or empiric findings as foundations in designing specific treatment programs for FD. To fill this knowledge gap, we designed a new psychotherapy.

From the Department of Psychology, The University of Hong Kong, Hong Kong (C.C.); the Institute of Basic Medicine, Capital University of Medical Sciences, Beijing, China (F.Y., S.J.); and South London and Maudsley NHS Trust, London, United Kingdom (J.M.H).

Address correspondence and reprint requests to Cecilia Cheng, $\mathrm{PhD}$, Department of Psychology, The University of Hong Kong, Pokfulam Road, Hong Kong. E-mail: ceci-cheng@hku.hk

Received for publication December 8, 2005; revision received July 21, 2006.

Preparation of this article was supported by the Hong Kong Research Grants Council's Competitive Earmarked Research Grant HKUST6047/02H and Direct Allocation Grant DAG03/04.HSS08 to the first author.

DOI: $10.1097 / 01 . p s y .0000249734 .99065 .6 f$
This psychotherapy differs from the existing FD therapies in its design, which is based on both the general cognitivebehavioral model and a body of studies on FD.

\section{Description of Flexible Coping Psychotherapy}

Previous studies $(6-8)$ have shown that patients with FD are characterized by an active inflexible coping style. Specifically, they tend to use problem-focused coping regardless of the controllability of stressful situations. Although problemfocused coping strategies are generally adaptive in mitigating anxiety and depression (e.g., $(9,10))$, these strategies can also magnify such psychological distress (e.g., $(8,11))$. On the other hand, studies $(6-8,12)$ revealed that emotion-focused coping, rather than problem-focused coping, is associated with less severe physical symptoms. We proposed the need to balance the active inflexible coping style of patients with FD by strengthening their use of emotion-focused coping strategies.

It is worth noting that the mere acquisition of skills may not necessarily lead to symptom relief, especially for long-term changes that last for more than 6 months after treatment. To foster long-term reduction in symptom severity, clients may have to understand how to a) deploy different strategies to cope with the specific demands of a variety of problems, and b) translate the skills learned in the treatment program into practical strategies for handling real-life problems. Previous studies on coping flexibility (13-15) revealed that problemfocused coping is more effective in mitigating distress in controllable situations, whereas emotion-focused coping is more effective in mitigating distress in uncontrollable situations. Apart from acquiring various types of fundamental skills (coping strategies) to handle stress, the flexible coping psychotherapy (FCP) advocates the acquisition of a "metaskill" of discriminative facility (see $(14,16)$ ). Specifically, this "metaskill" targets at a) sharpening their sensitivity in distinguishing among distinct demands of diverse situations, and b) increasing their flexibility to use distinct fundamental skills in different situations and symptom conditions.

\section{Overview of This Study}

This study was conducted to test the effectiveness of the proposed FCP. To identify which specific treatment compo- 


\section{CHENG et al.}

nents were responsible for symptom relief, we compared the FCP with supportive psychotherapy (SPP). Instead of including a no-treatment or waiting list control group, we chose SPP to control for the amount of time and attention spent on clients. A randomized, controlled design was adopted to compare the differences between the target treatment condition (FCP) and the control treatment condition (SPP). Each of these conditions comprises six 1-hour biweekly sessions over a period of 12 weeks.

The two treatment conditions were compared with examine whether the FCP would have therapeutic effects on coping flexibility and outcome changes independent of the time and attention spent with the clients. For the outcome analyses, the most common measure was self-rated dyspeptic symptom severity (SDSS; see e.g., $(17,18)$ ), and this variable was included as the a priori primary outcome variable. Secondary outcome variables include gastroenterologist-rated dyspeptic symptom severity (GDSS) and anxiety levels (see (19)). In this study, therapeutic effects were operationalized as a) increase in coping flexibility, b) reduction in SDSS and GDSS, and c) decrease in anxiety levels. The variables of coping flexibility, SDSS, GDSS, and anxiety levels were assessed before and 12 months after the psychotherapy.

\section{METHODS \\ Participants}

Figure 1 depicts the course of patients through the various phases of this study. We performed an a priori power test (20), which showed that at least 29 participants should be allocated into each of the two treatment conditions to yield $90 \%$ power ( $p=5 \%$, two-tailed). Taking both rejection and dropout rates into consideration, we initially recruited 150 Chinese adults with FD

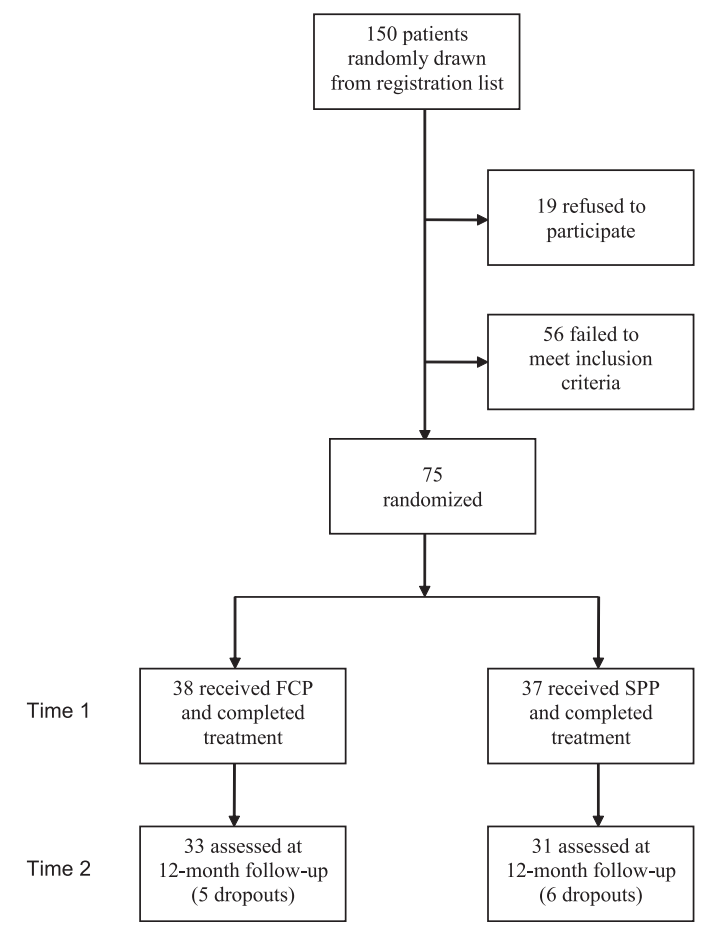

Figure 1. Flow diagram showing course of participants through various phases of the study. FCP = flexible coping psychotherapy; SPP = supportive psychotherapy. between August 2003 and September 2004. These potential participants were randomly chosen from a registration list of patients with FD recruited from the gastroenterology section of a university teaching hospital and a general hospital in Beijing, China.

All the participants met the Rome II Diagnostic Criteria (1) with dyspeptic symptoms for at least 12 weeks within 1 year but without endoscopic evidence of peptic ulcer disease, gastric cancer, gastritis, or esophagitis. These functional dyspeptic symptoms refer to epigastric pain and discomfort (e.g., belching, nausea, retching, vomiting). Also, they were diagnosed as endoscopically normal. Fifty-six patients were excluded because they met at least one of the following exclusion criteria: a) had received medical treatment for less than 3 years, b) had symptoms of irritable bowel syndrome, c) had heartburn or acid regurgitation, d) had undergone a surgery, e) had a history of serious physical and psychiatric illness, ${ }^{1}$ f) were pregnant, g) were under 18 year old, and h) were unwilling to give written informed consent.

Eligible participants were assigned to a treatment condition through concealed allocation. An independent research assistant, who was not directly involved in the conduct of various phases of the study, carried out the allocation process. Each of the 75 participants who met the inclusion criteria was assigned a number randomly generated by a computer program. Then the research assistant performed the randomization through which 38 participants were assigned to the target treatment condition and 37 were assigned to the control condition. Before participants signed the consent form, all were informed that the treatment had not yet been tested, so it might or might not be beneficial, and the sessions would be audiotaped to ensure the quality of treatment. The protocol of this study obtained prior approval from the Human Subject Research Panel of the university with which the first author was affiliated.

Eleven participants, five from the target group and six from the control group, dropped out (see Fig. 1). Participants who completed both phases of the study and those who dropped out in the second phase did not differ in any of the time 1 measures $(F(3,71)=0.36, p=.78)$.

For the final sample, the target group comprised 33 participants (12 males, 21 females), and the control group contained 31 participants (9 males, 22 females). The average age in the target and the control groups was 39.06 years (standard deviation $[\mathrm{SD}]=11.25$, range $=21-62$ years) and 41.81 years $(\mathrm{SD}=13.09$, range $=18-65$ years), respectively. For the target group, $7 \%$ had less than a high school education, $59 \%$ were high school graduates, and $34 \%$ were undergraduates or university graduates. For the control group, $8 \%$ had less than a high school education, $62 \%$ were high school graduates, and $30 \%$ were undergraduates or university graduates. Participants of the two groups did not statistically differ in the distribution of $\operatorname{sex}\left(\chi^{2}(1)=0.16, p=\right.$ $.69)$, age group $\left(\chi^{2}(4)=2.00, p=.74\right)$, and education level $\left(\chi^{2}(3)=3.11\right.$, $p=.38$ ). The two groups also did not statistically differ in the number of years of having $\mathrm{FD}(F(1,73)=0.60, p=.44$; mean $=4.87$ and $\mathrm{SD}=1.96$ for the FCP group; mean $=5.25$ and $\mathrm{SD}=2.26$ for the SPP group). All participants underwent a six-session psychotherapy program with the same therapist. They met the therapist individually for 1 hour biweekly for 12 weeks.

The multivariate analysis of variance (MANOVA) results revealed that none of the main and interaction effects among the demographic variables were statistically significant ( $F$ values $<1.01, p$ values $>.45$ ). The demographic variables were thus excluded in the subsequent statistical analyses.

\section{Treatment Conditions}

The FCP was adapted from a manualized model for patients with FD (21). The aims, framework, and scripts of each psychotherapy session were written in the manual and the therapist was advised to refer closely to the manual for standardization of practice. The first session included a) psychoeducation about the prevalence and nature of FD, and b) discussion about sources of stress and how they influence dyspeptic symptoms. Participants were then taught a variety of problem-focused and emotion-focused coping strategies in the next three sessions. In the subsequent sessions, participants were taught

\footnotetext{
${ }^{1}$ Serious physical and psychiatric illness refers to any illnesses that require medical attention for over 6 months.
} 
the "metaskill" of discriminative facility, that is, how to a) distinguish among the distinct characteristics of diverse stressful situations, and b) flexibly deploy various strategies learned in the previous sessions to handle life stress with specific demands and distinct levels of symptom severity. For instance, participants were taught to distinguish the extent of controllability of different stressful situations, and to deploy a) problem-focused coping when the outcome of a situation was deemed controllable and b) emotion-focused coping when the outcome was deemed uncontrollable. Participants were asked to practice these techniques and skills at home and to note any difficulties in using the techniques and skills on their own. Such difficulties in application were discussed in the next session.

The SPP (see (22)) was adopted to control the time, attention, and sympathy received by the participants in the target group. The contact time of this control treatment matched exactly that of the target treatment. In each psychotherapy session, participants were asked to express their feelings and distress related to their functional dyspeptic symptoms. They also received information about the prevalence and nature of FD. The therapist was instructed not to use any of the specific elements of the FCP in the control psychotherapy sessions.

\section{Therapist}

The psychotherapy sessions were conducted by a certified clinical psychologist with 4-year postdoctoral clinical experience. This therapist has received training in both cognitive-behavioral and person-centered psychotherapies, and believed that both types of psychotherapy were equally valid and useful to patients with functional dyspepsia. The same clinical psychologist delivered the psychotherapy for both FCP and SPP to eliminate the possibility of treatment differences resulting from different therapist effects. The sessions were supervised by an experienced clinical psychologist on a regular basis, and the amount and process of supervision were identical for the FCP and SPP sessions. All the sessions were audiotaped and randomly selected for reviews to ensure standardization of practice and fulfillment of ethical requirements.

\section{Therapist Adherence to Treatment Condition}

One session was randomly selected from each participant's psychotherapy and was rated by two independent raters to check whether the therapist's practice had adhered to a specific treatment condition specified by the psychotherapy manual. The kappa (23), a common measure of interrater agreement, showed good agreement among the raters $(\kappa=0.88)$. Ninety-six percent of the rated sessions were correctly assigned to the treatment condition from which they were drawn. The wrongly allocated sessions were typically found in the first session for which the two treatment conditions were very similar.

\section{Measures}

In this study, a set of questionnaires, which comprises the Cognitive Flexibility Questionnaire (CFQ), Dyspeptic Symptom Questionnaire (DSQ), and State-Trait Anxiety Inventory (STAI), was given to each participant before (time 1) and 12 months after (time 2) the treatment. These measures were administered by a research nurse at each time point. The therapist, supervisor, and gastroenterologists did not have access to the participants' data.

The CFQ (13) was used to examine coping flexibility. Participants were instructed to describe two controllable and two uncontrollable hassling events experienced in the past 6 months. They were then asked to report all the strategies deployed to handle each event and then to classify each strategy into one of two goals: "strategy used for managing the event" (i.e., problemfocused) and "strategy used for regulating the emotion associated with the event" (i.e., emotion-focused). Coping flexibility is operationalized by a strategy-situation fit index, which reflects the extent of situation-appropriateness of coping patterns. The scoring criterion derived from the goodness-of-fit theories $(24,25)$ was adopted. A score of 1 was given to the use of a) problem-focused coping in a controllable stressful situation, or b) emotionfocused coping in an uncontrollable stressful situation. A score of 0 was given to the use of a) problem-focused coping in an uncontrollable stressful situation, or b) emotion-focused coping in a controllable stressful situation. Be- cause there are considerable individual differences in the number of coping strategies used, the scores were aggregated and then divided by the total number of coping strategies. The index ranges from 0 (not flexible at all) to 1 (extremely flexible). The CFQ displayed good reliability, criterion-related validity, and discriminant validity $(13,14,26)$.

The DSQ $(26,27)$ was used to assess both SDSS and GDSS. This questionnaire assesses four main symptoms (i.e., epigastric pain, belching, nausea, and vomiting) commonly reported by patients with FD (27). Respondents rated the perceived severity of each symptom along an 11-point scale, which ranges from 0 (not severe at all) to 10 (extremely severe). The four items were aggregated to form a symptom severity score, which ranges from 0 to 40 . A higher score indicates a higher level of SDSS. This measure was found to be reliable and has good discriminant validity among patients with FD in Hong Kong $(8,26-28)$.

The DSQ was completed by not only the participants, but also gastroenterologists. Two gastroenterologists, each with more than 5 years of clinical experience, rated the questionnaire independently to yield the GDSS scores. Both of them have seen the participants for more than 2 years. Interrater agreement was high $(\kappa=0.91)$. This measure has good internal consistency and discriminant validity among Chinese patients with FD $(6,27)$. In this study, both self- and gastroenterologist ratings displayed good internal consistency (Cronbach $\alpha=0.83$ and 0.85).

The STAI $(29,30)$ was used to assess levels of anxiety. The T-Anxiety subscale, which comprises 20 statements, was adopted to measure trait anxiety. The anxiety scores range from 20 to 80 with a higher score indicating a higher trait anxiety level. This measure has been found reliable and valid in Chinese samples $(29,31)$. The items were also found to be internally consistent in this study (Cronbach $\alpha=0.91)$.

\section{Blinding}

Blinding was carried out at four different levels: a) the therapist who delivered the psychotherapy sessions and research nurse who delivered the questionnaire; b) gastroenterologists and endoscopists who performed the endoscopy and made diagnoses; c) research assistants who performed adherence ratings of psychotherapy sessions, data entry, and statistical analyses; and d) participants with FD. The therapist and research nurse were blind to the research hypotheses, participants' medical histories, and participants' selfrated symptoms. The gastroenterologists and endoscopists were blind to the research hypotheses, the treatment condition to which participants were assigned, and participants' self-rated symptoms. The research assistants were blind to the research hypotheses, the treatment condition to which participants were assigned, and participants' medical histories. The participants were blind to the research hypotheses and the treatment condition to which they were assigned.

\section{Procedure}

Participants had to give informed consent before the study began. After giving written consent, participants completed a set of questionnaires distributed by a research nurse. At the follow-up phase, the same set of questionnaires was mailed to participants, and a return envelope was provided for the completed questionnaires. Reminder notes were sent to those participants who did not return the questionnaire within 2 and 4 weeks, respectively. A debriefing note was sent to all participants after they had returned the questionnaires. Participants in the SPP condition were invited to take part in an upcoming FCP if interested.

\section{Statistical Analyses}

Before performing the main analyses, we first examined the interrelationships among the variables with Pearson product-moment correlation coefficients. Then we scrutinized longitudinal changes in coping flexibility and outcome variables between the groups over the 12-month period. We adopted a 2 (group: target versus control) $\times 2$ (time: pre- versus posttreatment) mixed-design (split plot) MANOVA with group as a between-participants variable and time as a repeated measure. A significant group $\times$ time interaction indicates that the extent of changes in a variable is statistically different between the two treatment groups. 
Apart from examining statistical effects of treatment outcomes, clinically significant changes should also be evaluated. Two sets of clinical significance analyses were conducted to assess the practical value regarding the clinical effects of a treatment. The first set identifies the level of changes induced by a treatment. Reliable change index (RCI) (32) was calculated for this purpose because this method of comparison has been found valid in distinguishing reliable from unreliable changes in treatment outcomes (e.g., $(33,34)$ ).

The second set assesses the clinical meaningfulness of the changes in the primary outcome, which is operationalized by equivalent posttreatment SDSS scores between a treatment group after attending psychotherapy and a nonclinical group. Normative comparisons (35) were performed because these procedures provide a robust test of treatment efficacy by a norm or criterion independent of the clinical samples.

\section{RESULTS}

\section{Relationships Between Coping Flexibility and Outcome Measures}

Table 1 shows that the correlation coefficients among the study variables. Time 1 coping flexibility was inversely related to time 1 anxiety and time 2 anxiety. Time 2 coping flexibility was also inversely related to time 2 SDSS and time 2 anxiety. Although these results suggest that greater flexibility in coping is related to reductions in FD symptoms, it is worth noting that coping flexibility was unrelated to SDSS at time 1. The association between coping flexibility and FD symptoms was statistically significant only after the participants had received psychotherapy.

Additional correlational analyses were conducted to examine changes in coping flexibility and changes in symptoms over time. Results showed that changes in coping flexibility were inversely related to both changes in SDSS and changes in GDSS $(r \mathrm{~s}(64)=-0.39$ and $-0.37, p$ s $<.002)$. The inverse association between changes in coping flexibility and changes in anxiety was not statistically significant $(r(64)=$ $-0.20, p=.11$ ).

\section{Longitudinal Analyses of Changes in Coping Flexibility}

Table 2 presents descriptive statistics for each time point for the group of participants who received FCP (treatment group) and the group of participants who received SPP (control group). For coping flexibility, the MANOVA results showed that the main effects of group and time were both statistically significant $(F$ values $(1,62)=8.38$ and 9.51, mean standard errors $[\mathrm{MSEs}]=0.10$ and $0.08, p$ values $<.003$ ). These statistically significant main effects should be interpreted in light of the statistically significant interaction between group and time $(F(1,62)=6.80, \mathrm{MSE}=0.08, p=$ $.01)$. Such results indicate that the target group displayed greater flexibility in coping, but the control group showed no changes in coping flexibility from time 1 to time 2 .

\section{Longitudinal Analyses of Outcome Changes}

For SDSS, there were statistically significant main effects of group and time (F values $(1,62)=48.40$ and 457.10, MSEs $=21.91$ and $10.80, p$ values <.0001). The group $\times$ time interaction effect was also statistically significant $(F(1$, $62)=68.61, \operatorname{MSE}=10.80, p<.0001)$. From time 1 to time 2 , both the target and the control groups reported a reduction in SDSS, but the target group reported a greater extent of reduction than did the control group.

For GDSS, results revealed statistically significant main effects of group and time $(F$ values $(1,62)=23.65$ and 197.75, MSEs $=25.39$ and $7.38, p$ values $<.0001)$. The group $\times$ time interaction effect was statistically significant $(F(1,62)=62.18, \mathrm{MSE}=7.38, p<.0001)$. Such results indicate that there was a decrease in GDSS for both the target and the control groups, but the extent of reduction was greater for the target group.

For anxiety, the main effect of time was statistically significant $(F(1,62)=482.94$, MSE $=45.87, p<.0001)$. This result indicates that from time 1 to time 2 , both groups reported a similar reduction in anxiety levels.

\section{Clinical Significance of Change Reliable Change Identification}

The RCI was calculated to indicate the amount of change that occurred after a treatment for each of the outcome measures. A value greater than or equal to 1.96 indicates a reliable change in treatment outcomes (see (32)). The right panel of Table 1 summarizes the RCI for each treatment outcome for the two treatment groups. The FCP produced reliable changes in the treatment outcomes. The SPP also produced reliable changes in the treatment outcomes with the exception of GDSS.

TABLE 1. Zero-Order Correlation Coefficients for Study Variables $(N$ for time $1=75, N$ for time $2=64)$

\begin{tabular}{|c|c|c|c|c|c|c|c|}
\hline & 2 & 3 & 4 & 5 & 6 & 7 & 8 \\
\hline 1. Coping flexibility_time 1 & -0.14 & -0.01 & $-0.50^{b}$ & 0.12 & -0.03 & 0.19 & $-0.31^{\circ}$ \\
\hline 2. SDSS-time 1 & - & $0.47^{b}$ & $0.53^{b}$ & 0.21 & $0.31^{a}$ & $0.52^{b}$ & $0.44^{b}$ \\
\hline 3. GDSS-time 1 & & - & $0.26^{a}$ & 0.19 & 0.18 & $0.43^{b}$ & $0.26^{\circ}$ \\
\hline 4. Anxiety time 1 & & & - & $-0.17^{a}$ & -0.12 & $0.42^{b}$ & $0.66^{b}$ \\
\hline 5. Coping Flexibility-time 2 & & & & - & $-0.44^{b}$ & -0.15 & $-0.31^{b}$ \\
\hline 6. SDSS-time 2 & & & & & - & $0.62^{b}$ & 0.10 \\
\hline 7. GDSS-time 2 & & & & & & - & $0.30^{\circ}$ \\
\hline 8. Anxiety-time 2 & & & & & & & - \\
\hline
\end{tabular}

${ }^{a} p<.05$.

SDSS = self-rated dyspeptic symptom severity; GDSS = gastroenterologist-rated dyspeptic symptom severity. 
TABLE 2. Descriptive Statistics and Reliable Change Index for Study Variables

\begin{tabular}{|c|c|c|c|c|c|c|c|c|}
\hline & \multirow{2}{*}{ Treatment Group } & \multicolumn{3}{|c|}{$\begin{array}{l}\text { Time } 1 \\
(N=75)\end{array}$} & \multicolumn{3}{|c|}{$\begin{array}{l}\text { Time } 2 \\
(N=64)\end{array}$} & \multirow{2}{*}{$\mathrm{RCl}$} \\
\hline & & Mean & SD & $\begin{array}{l}95 \% \text { Confidence } \\
\text { Interval }\end{array}$ & Mean & SD & $\begin{array}{l}\text { 95\% Confidence } \\
\text { Interval }\end{array}$ & \\
\hline \multirow[t]{2}{*}{ Coping flexibility } & Flexible coping psychotherapy & 0.27 & 0.32 & $(0.16-0.38)$ & 0.56 & 0.35 & $(0.46-0.66)$ & $2.36^{a}$ \\
\hline & Supportive psychotherapy & 0.24 & 0.31 & $(0.13-0.36)$ & 0.27 & 0.21 & $(0.16-0.37)$ & 0.08 \\
\hline \multirow[t]{2}{*}{ SDSS } & Flexible coping psychotherapy & 28.64 & 3.38 & $(27.31-29.96)$ & 11.39 & 4.03 & $(9.91-12.88)$ & $8.92^{a}$ \\
\hline & Supportive psychotherapy & 29.58 & 4.22 & $(28.21-30.95)$ & 21.97 & 4.51 & $(20.44-23.50)$ & $2.83^{a}$ \\
\hline \multirow[t]{2}{*}{ GDSS } & Flexible coping psychotherapy & 25.45 & 3.99 & $(24.04-26.87)$ & 14.91 & 4.01 & $(13.51-16.31)$ & $4.25^{a}$ \\
\hline & Supportive psychotherapy & 26.00 & 4.16 & $(24.54-27.46)$ & 23.03 & 4.04 & $(21.59-24.48)$ & 1.27 \\
\hline \multirow[t]{2}{*}{ Anxiety } & Flexible coping psychotherapy & 58.30 & 11.54 & $(54.38-62.23)$ & 32.36 & 13.16 & $(28.22-36.51)$ & $5.51^{a}$ \\
\hline & Supportive psychotherapy & 58.13 & 10.99 & $(54.08-62.18)$ & 31.42 & 10.44 & $(27.14-35.70)$ & $5.75^{a}$ \\
\hline
\end{tabular}

${ }^{a}$ Reliable changes over time.

$\mathrm{RCI}=$ reliable change index; SDSS = self-rated dyspeptic symptom severity; GDSS = gastroenterologist-rated dyspeptic symptom severity.

\section{Normative Comparisons}

Normative comparisons evaluate the efficacy of a treatment in relieving dyspeptic symptoms with a criterion independent of clinical samples. However, no published norms were available for the outcome measure of dyspeptic symptom severity. Following the suggestions by Kendall and colleagues, we used data drawn from a community sample of healthy Chinese as reported in another study (6). In that study, 83 Chinese healthy participants, defined as having no major health problems within the past 5 years, completed the same measure. The mean SDSS score of this sample was $7.65(\mathrm{SD}=8.78)$. Kendall and colleagues recommended the adoption of $1 \mathrm{SD}$ $(\delta)$ for defining the range of closeness within which a treat- ment group and the nonclinical group is deemed clinically equivalent. The upper bound of the normative range was 16.43 (i.e., $7.65+\delta$ ). Figure 2 depicts the SDSS scores for the FCP and SPP groups across the two phases of this study along with the normative range for normative comparisons.

After specifying the range of closeness, we conducted both a clinical equivalency test and a traditional $t$ test (i.e., independent-samples $t$ test) to determine whether a treatment produced changes such that clients who received treatment are similar to the nonclinical norms. The clinical equivalency test examines whether the mean difference between a treatment group and the healthy community group lies within the specified range of closeness. Results showed that the difference

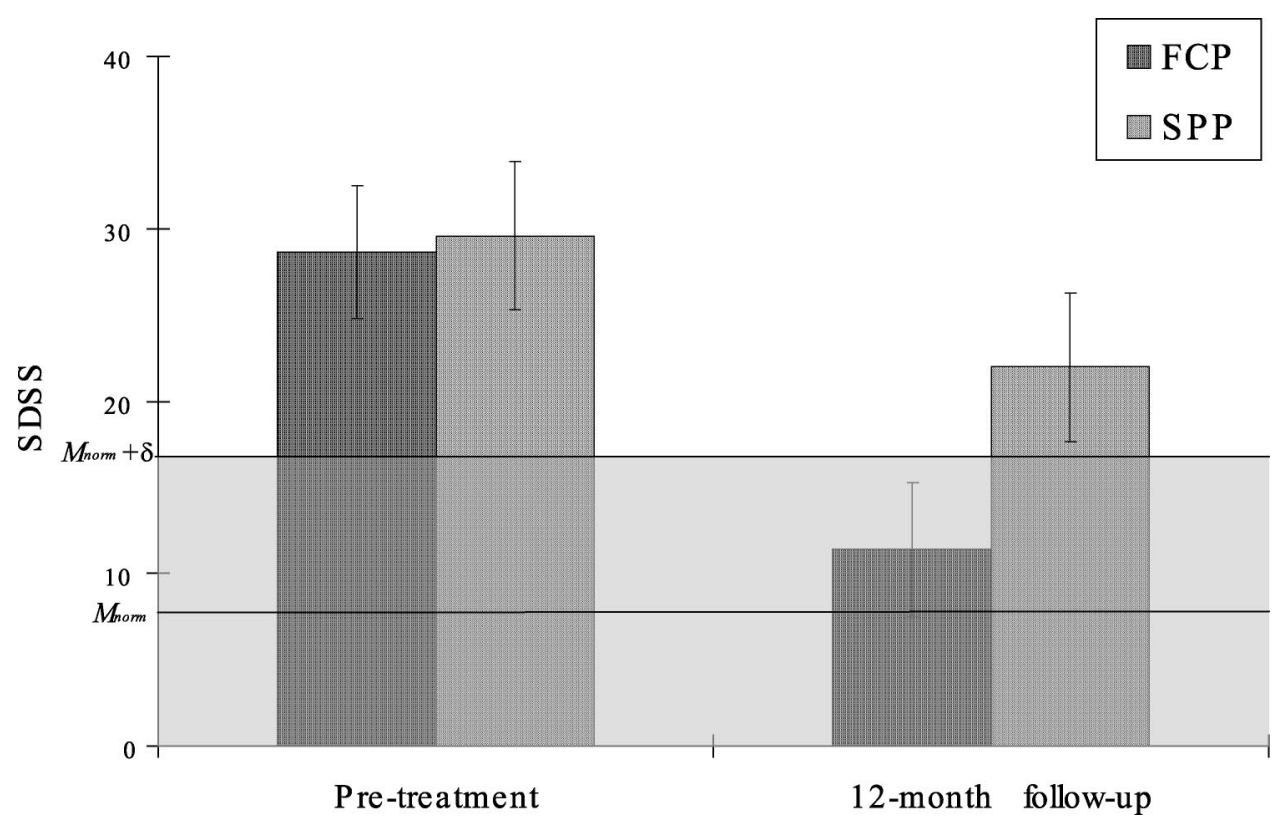

Figure 2. Self-rated dyspeptic symptom severity (SDSS) assessed before and after treatment for the flexible coping psychotherapy (FCP) and supportive psychotherapy (SPP) groups. Bars indicate $95 \%$ confidence intervals. $M_{\text {norm }}$ indicates normative mean. The gray shaded area shows the normative range of scores drawn from a community sample of healthy Chinese individuals. 


\section{CHENG et al.}

between the mean score of the FCP group assessed at time 2 and the normative mean fell within the range of closeness $($ C.E. $t(114)=3.82, p<.0001)$.

The independent-samples $t$ test examines whether the mean of a treatment group and that of the healthy community group are statistically different from each other. Results showed no statistically significant differences between the mean score of the FCP group at time 2 and that of the healthy community group $(t(114)=1.09, p=.28)$. According to Kendall and colleagues, clinical equivalence is indicated by both a significant $t$ value in the clinical equivalency test and a nonsignificant $t$-value in the independent-samples $t$ test. Meeting these criteria, these two results suggested that only the FCP produces a practically significant reduction in SDSS 12 months after treatment. Taken together, the present results provide support for the effectiveness of FCP in producing a reliable reduction in dyspeptic symptoms for patients with FD.

\section{DISCUSSION}

The present study may contribute to the extant literature in several ways. Specifically, this study was the first to use a FCP for enhancing the coping flexibility of patients with FD. The components of this psychotherapy are derived from previous findings on FD. More broadly, this project lays out a systematic structure through the combination of empiric findings and techniques. Adopting this new psychotherapy, the present study provided tentative support that the FCP produces both statistically and clinically significant changes in FD symptom severity.

SPP is currently the most popular approach adopted in psychological intervention programs for treating FD (22). It advocates the reduction of symptoms through the provision of support to empower clients and education materials to enhance clients' understanding of their own health conditions. While providing the same amount of support and education materials, the FCP also emphasizes acquisition of techniques and, more importantly, flexible deployment of distinct techniques to handle specific demands of different stressful situations and symptom conditions (see $(13,14)$ ).

The present study revealed that both the FCP and the SPP were beneficial in bringing about desirable outcomes for patients with FD. A comparison of the outcomes yielded by these psychotherapy programs may foster the identification of the specific elements of psychotherapy that produce positive changes in clients. To elaborate, the FCP and the SPP are both effective in anxiety reduction. Because both psychotherapies render social support and education materials, the present results suggest that these two social support elements are related to long-term changes in anxiety levels.

Compared with the SPP, however, the FCP was found to be more efficacious in reducing FD symptoms. Only the clients receiving FCP reported clinical reductions in dyspeptic symptom severity. It is worth noting that such a clinical significance of change was found to be present 12 months after the psychotherapy program. Such a finding suggests that the techniques learned from the $\mathrm{FCP}$ - especially for sensitivity in distinguishing among distinct stressful events and flexible application of diverse strategies to handle distinct stressful situations - can be implemented and integrated into one's daily life.

Apart from the SPP, medical intervention is also commonly adopted for treatment of FD, a common functional gastroenterological disorder. Functional gastroenterological disorders impose a substantial economical burden in terms of medical consultation and drug costs (36). According to a large-scale population-based survey on more than 5500 adults across seven regions of the world (37), 30\% of individuals with functional gastroenterological disorders had taken either overthe-counter or prescribed drugs. One percent of them reported having stayed at a hospital for their problems. In the United States, the annual medical and drug cost of functional gastroenterological disorders was an estimated $\$ 9.3$ billion (38). Because the FCP was found to be useful in producing positive therapeutic outcomes, the results may have implications for primary practice as an alternative to current medical treatment, thus reducing drug consumption and the financial burden on primary care services. Future studies should be conducted to examine possible differences in effectiveness and duration between the FCP and medical intervention.

Before concluding, several caveats are worth noting. This study is an initial attempt to examine the efficacy of a new psychotherapy on treating FD symptoms and thus the present findings should be regarded as tentative. First, the potential problem related to the generalizability of findings should be noted. Specifically, the present sample was limited to patients with FD who attended the outpatient section of two public hospitals. Because the presentation of somatic symptoms for patients who attended walk-in clinics differ from that of patients who attended private clinics with personal physicians (39), future studies should examine the generalizability of the results to patients from a variety of clinic settings, especially those from private clinics. Also, participants in this study were all having FD symptoms for at least 3 years. Further studies should explore the effectiveness of FCP to more heterogeneous samples such as those with a shorter history of having FD.

Second, it is important to note that participants in this study were all ethnically Chinese. Previous studies $(40,41)$ have shown that the prevalence rates of having functional gastrointestinal disorders, the physiological mechanisms influencing gastrointestinal symptoms, and the psychological profiles of Chinese patients with functional gastrointestinal disorders were highly similar to patients from Western countries. However, the study by Hsu and Folstein (42) revealed that Chinese American patients tended to report more abnormal sensations, whereas white patients tended to report more abnormal motor functions. This study should thus be replicated in other cultural settings, preferably by crosscultural studies, to address the issue concerning the generalizability of the present results across cultures.

Third, although the power test showed that the sample size was adequate, the number of participants in this sample was 
relatively small compared with the population of Chinese patients. Further studies with a larger sample drawn from a variety of Chinese populations (e.g., both urban and rural areas) should be conducted to evaluate whether the present psychotherapy has the potential to relieve the symptoms of FD in a diverse group of patients. Moreover, a larger sample size enables researchers to compare the efficacy of the FCP among patients with FD with distinct predominant gastrointestinal problems (e.g., epigastric pain, belching).

Fourth, it is worth noting that the follow-up phase was conducted 12 months after the participants had received psychotherapy. A limitation of the design of this study is that intermediate time points were lacking and thus the short-term effects of the FCP were unknown. Multiple-phase studies should be conducted to include more time points (such as immediately after psychotherapy, 3-month follow up) to examine immediate, short-term, and long-term effectiveness of the FCP on FD.

To conclude, FD was once a conundrum to researchers, who attempted to relate the disorder to certain pathogenic physical mechanisms but failed (see (43)). The growing interest in exploring psychological factors has led to studies that enhance the understanding of the mechanisms that influence symptom severity of the disorder. The present study may provide further insights on possible reductions in FD symptoms through the adoption of the FCP.

We thank Jing Chen, Gigi Lam, Yuen-yan Law, Kit-yan Lee, Gang Lu, Guoxin Meng, Xiulin Sun, Mui-fan Yong, and Xiaopeng Zhang for research assistance.

\section{REFERENCES}

1. Drossman DA, Corazziari ES, Talley NJ, Thompson WG, Whitehead WE. Rome II: The Functional Gastrointestinal Disorders: Diagnosis, Pathophysiology, and Treatment: A Multinational Consensus. McLean, VA: Degnon Associates, 2000.

2. Talley NJ, Axon A, Bytzer P, Holtmann G, Lam SK, Van Zanten S. Management of uninvestigated and functional dyspepsia: a Working Party report for the World Congresses of Gastroenterology 1998. Aliment Pharmacol Ther 1999;13:1135-48.

3. Forbes A, MacAuley S, Chiotakakou-Faliakou E. Hypnotherapy and therapeutic audiotape: effective in previously unsuccessfully treated irritable bowel syndrome? Int J Colorectal Dis 2000;15:328-34.

4. Dotevall G. Functional dyspepsia: physiology, diagnosis and treatment. Stress Med 1994;10:101-5.

5. Kellner R. Psychosomatic syndromes, somatization, and somatoform disorders. In: Fava GA, Freyberger H, eds. Handbook of Psychosomatic Medicine. Madison, CT: International Universities, 1998:125-60.

6. Cheng C, Hui W, Lam S. Coping style of individuals with functional dyspepsia. Psychosom Med 1999;61:789-95.

7. Cheng C, Hui W, Lam S. Coping with first-time endoscopy for a select sample of Chinese patients with functional dyspepsia and duodenal ulcer: an observation study. Psychosom Med 2002;64:867-73.

8. Cheng C, Hui W, Lam S. Perceptual style and behavioral pattern of individuals with functional gastrointestinal disorders. Health Psychol 2000;19:146-54.

9. Kim KI, Won H, Liu X, Liu P, Kitanishi K. Students' stress in China, Japan and Korea: a transcultural study. Int J Soc Psychiatry 1997;43: $87-94$.

10. Marx EM, Schulze CC. Interpersonal problem-solving in depressed students. J Clin Psychol 1991;47:361-7.

11. Folkman S, Lazarus RS, Gruen RJ, DeLongis A. Appraisal, coping, health status, and psychological symptoms. J Pers Soc Psychol 1986;50: $571-9$.
12. Levenson JL, Mishra A, Hamer RM, Hastillo A. Denial and medical outcome in unstable angina. Psychosom Med 1989;51:27-35.

13. Cheng C. Assessing coping flexibility in real-life and laboratory settings: a multimethod approach. J Pers Soc Psychol 2001;80:814-33.

14. Cheng C. Cognitive and motivational processes underlying coping flexibility: a dual-process model. J Pers Soc Psychol 2003;84:425-38.

15. Schwartz CE, Peng CK, Lester N, Daltroy L, Goldberger AL. Selfreported coping behavior in health and disease: assessment with a card sort game. Behav Med 1998;24:41-4.

16. Cheng C, Chiu C, Hong Y, Cheung JS. Discriminative facility and its role in the perceived quality of interactional experiences. J Pers 2001;69: $765-86$.

17. Jiang B, Lin J, Zhang Y. Clinical observation on the mental symptoms of functional dyspepsia and its treatment [in Chinese]. Zhongguo Zhong Xi Yi Jie He Za Zhi 2000;20:424-6.

18. Mine K, Kanazawa F, Hosoi M, Kinukawa N, Kubo C. Treating nonulcer dyspepsia considering both functional disorders of the digestive system and psychiatric conditions. Dig Dis Sci 1998;43:1241-7.

19. Rentz AM, Battista C, Trudeau E, Jones R, Robinson P, Sloan S, Mathur S, Frank L, Revicki DA. Symptom and health-related qualityof-life measures for use in selected gastrointestinal disease studies: a review and synthesis of the literature. Pharmacoeconomics 2001;19: $349-63$.

20. Cohen J. Statistical Power Analysis for the Behavioral Sciences, 2nd ed. Hillsdale, NJ: Lawrence Erlbaum Associates, 1988.

21. Cheng C, Hutton JH. Manual of Multiple-Component Psychotherapy for Functional Dyspeptic Patients. Hong Kong: Hong Kong University of Science and Technology, 2003.

22. Freyberger H, Freyberger HJ. Supportive psychotherapy. In: Fava GA, Freyberger H, eds. Handbook of Psychosomatic Medicine. Madison, CT: International Universities, 1998:639-56.

23. Cohen J. A coefficient of agreement for nominal scales. Educ Psychol Meas 1960;20:37-46.

24. Aldwin CM. Stress, Coping, and Development: An Integrative Perspective. New York: Guilford Press, 1994.

25. Miller SM. Individual differences in the coping process: what to know and when to know it. In: Carpenter BN, ed. Personal Coping: Theory, Research, Application. Westport, CT: Praeger Publishers, 1992:77-91.

26. Cheng C, Hui W, Lam S. Psychosocial factors and perceived severity of functional dyspeptic symptoms: a psychosocial interactionist model. Psychosom Med 2004;66:85-91.

27. Hu WHC, Lam KF, Lam CLK, Wong NYH, Hui W, Lam S. A questionnaire to assess symptom severity in patients with functional dyspepsia. Gastroenterology 1999;116:A1010.

28. Cheng C. Gender-role differences in susceptibility to the influence of support availability on depression. J Pers 1999;67:439-67.

29. Shek DTL. Reliability and factorial structure of the Chinese version of the State-Trait Anxiety Inventory. J Psychopathol Behav Assess 1988; 10:303-17.

30. Spielberger CD. Manual for the State-Trait Anxiety Inventory (Form Y). Palo Alto, CA: Consulting Psychologists Press, 1983.

31. Cheng C. Seeking medical consultation: perceptual and behavioral characteristics distinguishing consulters and nonconsulters with functional dyspepsia. Psychosom Med 2000;62:844-52.

32. Jacobson NS, Truax P. Clinical significance: a statistical approach to defining meaningful change in psychotherapy research. In: Kazdin AEE, ed. Methodological Issues and Strategies in Clinical Research. Washington, DC: American Psychological Association, 1992:631-48.

33. Ankuta GY, Abeles N. Client satisfaction, clinical significance, and meaningful change in psychotherapy. Prof Psychol Res Pr 1993;24: $70-4$.

34. Lunnen KM, Ogles BM. A multiperspective, multivariable evaluation of reliable change. J Consult Clin Psychol 1998;66:400-10.

35. Kendall PC, Marrs-Garcia A, Nath SR, Sheldrick RC. Normative comparisons for the evaluation of clinical significance. J Consult Clin Psychol 1999;67:285-99.

36. Drossman DA, Li Z, Andruzzi E, Temple RD, Talley NJ, Thompson WG, Whitehead WE, Janssens J, Funch-Jensen P, Corazziari E, Richter JE, Koch GG. US householder survey of functional gastrointestinal disorders: prevalence, sociodemography, and health impact. Dig Dis Sci 1993;38:1569-80. 


\section{CHENG et al.}

37. Haycox A, Einarson T, Eggleston A. The health economic impact of upper gastrointestinal symptoms in the general population: results from the Domestic/International Gastroenterology Surveillance Study (DIGEST). Scand J Gastroenterol 1999;Suppl 231:38-47.

38. Sandler RS, Everhart JE, Donowitz M, Adams E, Cronin K, Goodman C, Gemmen E, Shah S, Avdic A, Rubin R. The burden of selected digestive diseases in the United States. Gastroenterology 2002;122:1500-11.

39. Simon GE, VonKorff M, Piccinelli M, Fullerton C, Ormel J. An international study of the relation between somatic symptoms and depression. N Engl J Med 1999;341:1329-35.

40. Hu WHC, Hui WM, Lam CLK, Lam SK. Anxiety and depression are co-factors determining health care utilisation in patients with dyspepsia: a Hong Kong population based study. Gastroenterology 1997;112: A153.

41. Lee CT, Chuang TY, Lu CL, Chen CY, Chang FY, Lee SD. Abnormal vagal cholinergic function and psychological behaviors in irritable bowel syndrome patients: a hospital-based oriental study. Dig Dis Sci 1998;43: 1794-9.

42. Hsu LK, Folstein MF. Somatoform disorders in Caucasian and Chinese Americans. J Nerv Ment Dis 1997;185:382-7.

43. Witteman EM, Tytgat GN. Functional dyspepsia. Neth J Med 1995;46: 205-11. 